\title{
openheart A zero coronary artery calcium score in patients with stable chest pain is associated with a good prognosis, despite risk of non-calcified plaques
}

\author{
Xue Wang, ${ }^{1}$ Elizabeth Phuong Vi Le, ${ }^{1}$ Nikil K Rajani, ${ }^{2}$ NJ Hudson-Peacock, \\ Holly Pavey, ${ }^{3}$ Jason M Tarkin, ${ }^{1}$ Judith Babar, ${ }^{4}$ Michelle Claire Williams, ${ }^{5}$ \\ Deepa Gopalan, ${ }^{4}$ James H F Rudd ${ }^{1}$
}

To cite: Wang X, Le EPV, Rajani NK, et al. A zero coronary artery calcium score in patients with stable chest pain is associated with a good prognosis, despite risk of noncalcified plaques. Open Heart 2019;6:e000945. doi:10.1136/ openhrt-2018-000945

Received 9 0ctober 2018 Revised 7 March 2019 Accepted 18 March 2019

Check for updates

(c) Author(s) (or their employer(s)) 2019. Re-use permitted under CC BY. Published by BMJ.

${ }^{1}$ Division of Cardiovascular Medicine, University of Cambridge, Addenbrooke's Hospital, Cambridge, UK ${ }^{2}$ Department of Clinical Radiology, Imperial College Hospitals NHS Trust, St Mary's Hospital, London, UK ${ }^{3}$ Cambridge Clinical Trials Unit, Cambridge University Hospitals NHS Foundation Trust, Addenbrooke's Hospital, Cambridge, UK

${ }^{4}$ Department of Radiology, Addenbrooke's Hospital, Cambridge, UK

${ }^{5}$ Centre for Cardiovascular Sciences, University of Edinburgh, Edinburgh, UK

Correspondence to Dr James H F Rudd, Division of Cardiovascular Medicine, University of Cambridge, Addenbrooke's Hospital, Cambridge CB2 0QQ, UK; jhfr2@ cam.ac.uk

\section{ABSTRACT}

Objectives To estimate the prevalence of non-calcified coronary artery disease (CAD) in patients with suspected stable angina and a zero coronary artery calcification (CAC) score, and to assess the prognostic significance of a zero CAC in these symptomatic patients.

Methods In this prospective cohort study, consecutive patients with stable chest pain underwent CAC scoring \pm CT coronary angiography (CTCA) as part of routine clinical care at a single tertiary centre over 7 years. Major adverse cardiac event (MACE) was defined as cardiac death, non-fatal myocardial infarction and/or non-elective revascularisation.

Results A total of 915 of 1753 (52.2\%) patients (mean age $56.8 \pm 12.0$ years; $46.2 \%$ male) had a zero CAC score. Of the $751(82.1 \%)$ patients with a zero CAC in whom CTCA was performed, 674 (89.7\%) had normal coronary arteries, $63(8.4 \%)$ had non-calcified CAD with $<50 \%$ stenosis and $14(1.9 \%)$ had $\geq 50 \%$ stenosis in at least one coronary artery. The negative predictive value of a zero CAC for excluding a $\geq 50 \%$ CTCA stenosis was $98.1 \%$. Over a median follow-up period of 2.2 years (range 1.0-7.0 years), the absolute annualised rates of MACE were as follows: zero CAC 1.9 per 1000 personyears and non-zero CAC 7.4 per 1000 person-years (HR $3.8, p=0.009$ ). However, after adjusting for age, gender and cardiovascular risk factors using a multivariable Cox proportional hazards model, there was no statistically significant difference in the risk of MACE between the two patient cohorts $(p=0.19)$. After adjusting for age, gender and cardiovascular risk factors, the HR for all-cause mortality among the zero CAC cohort vers non-zero CAC was $2.1(p=0.27)$.

Conclusion A zero CAC score in patients undergoing CT scanning for suspected stable angina has a high negative predictive value for the exclusion of obstructive $C A D$ and is associated with a good medium-term prognosis.

\section{INTRODUCTION}

Coronary artery calcification (CAC) is a well-established marker of future cardiovascular risk. ${ }^{1}$ Cardiac CT is widely used for the evaluation of stable coronary artery disease

\section{Key questions}

What is already known about this subject?

- Many existing studies have looked at the prognostic value of a zero calcium score in asymptomatic study populations which have different prior probabilities of risk of major adverse cardiac event compared with symptomatic patient populations.

What does this study add?

- This study has the advantage of being a prospective study and looks at the medium-term prognosis of the symptomatic patient population with a zero calcium score.

How might this impact on clinical practice?

- The discovery of underlying coronary artery disease in those with zero calcium scores highlights the advantages of using CT coronary angiography in all subjects rather than having calcium scoring as a gatekeeper, as was the case in the previous version of the National Institute for Health and Care Excellence CG95 guideline.

(CAD) due to it being a non-invasive, cost-effective and highly sensitive technique. ${ }^{2}$ CT calcium scoring detects and quantifies CAC and coronary CT coronary angiography (CTCA) allows for detailed anatomical evaluation of luminal stenosis secondary to both calcified and non-calcified atherosclerotic plaques. $^{2}$

The absence of CAC (defined as a zero CAC score on CT) in asymptomatic individuals is associated with a very low incidence of cardiovascular events over a 15-year follow-up. ${ }^{3}$ However, $1 \%-2 \%$ of symptomatic patients with chest pain, and a zero CAC score, have non-calcified coronary artery atherosclerosis. ${ }^{45}$ The long-term prognosis of these symptomatic patients with a zero CAC score remains unclear. ${ }^{6}$ 
In November 2016, the UK's National Institute for Health and Care Excellence's (NICE) Clinical Guideline 95 for the evaluation of patients with stable chest pain was issued as an update of their own 2010 Guideline. ${ }^{27}$ The update recommended the removal of CT calcium scoring from the investigation algorithm on the basis that a zero CAC score may still be associated with significant underlying $\mathrm{CAD}$, particularly non-calcified plaque, instead suggesting CTCA for all patients presenting with suspected angina.

Therefore, the objective of the study was to estimate the prevalence of non-calcified CAD in patients presenting with stable angina and a zero CAC score undergoing CT scanning in our centre. The secondary objectives were to compare incidence rates of major adverse cardiac events (MACEs), and all-cause mortality, in patients with a zero CAC versus a non-zero CAC score.

\section{METHODS}

\section{Study design}

In this prospective study, consecutive patients with a suspected new diagnosis of stable angina underwent CAC scoring \pm CT coronary angiography (CTCA) as part of routine clinical care at Addenbrooke's Hospital from November 2009 to October 2016. This was a service evaluation audit and it was approved by the hospital's audit committee. Patients were excluded from the study if they were asymptomatic, had prior diagnosis of $\mathrm{CAD}$, myocardial infarction, percutaneous coronary intervention or coronary artery bypass grafting surgery. Patients referred for a cardiac CT scan for non-coronary indications such as arrhythmias, aortic valve and pericardial evaluations were excluded.

Clinical data were obtained from the hospital electronic patient record system. Hypertension was defined by a physician diagnosis of hypertension or the use of anti-hypertensive medications. Diabetes was defined by a physician diagnosis of diabetes or the use of anti-diabetic medications. Obesity was defined by a body mass index of 30 or greater. A positive smoking history was defined by the patient either being a current smoker (being an active smoker at the time of scan) or being an ex-smoker. A family history of premature CAD was defined as any first-degree relative with a history of relevant heart disease before age 60 or based on having a recorded family history of CAD in the patient record.

\section{CT imaging}

Imaging was performed using a Siemens Flash $64 \times 2$-slice dual source CT scanner as per standard clinical protocols. CAC scoring was performed using the Agatston $\operatorname{method}^{8}$ with Siemens SyngoVia analysis software V.VB9 and V.VB10. Prior to CTCA imaging, intravenous metoprolol (5-20 mg) and sublingual glyceryl trinitrate (two sprays, $400 \mu \mathrm{g}$ ) were administered to all patients without contraindication. Intravenous contrast $(70 \mathrm{~mL}$ Omnipaque 350) was administered at $6 \mathrm{~mL} /$ second followed by $40 \mathrm{~mL} 0.9 \%$ saline flush. CTCA images were acquired with prospective ECG gating (70\%), high-pitch single heart beat acquisition, retrospective mode or a combination as needed to obtain diagnostic image quality. Tube current was 150-300 mA and voltage 80-100 kV. CAC scanning and CTCA were performed on the same day. All CT scans were reported jointly by accredited cardiac radiologists and cardiologists. The severity of non-calcified CAD on CTCA was graded as follows: normal coronary arteries, non-obstructive (at least one $<50 \%$ luminal diameter stenosis) and obstructive (at least one $\geq 50 \%$ luminal diameter stenosis).

\section{Patient follow-up}

Patients were followed up prospectively using the hospital electronic patient record system.

The endpoint, MACE, was defined as cardiac death, non-fatal myocardial infarction and/or non-elective revascularisation. Additionally, the incidence of all-cause mortality was evaluated. Revascularisation was defined as percutaneous coronary intervention or coronary artery bypass grafting surgery. A diagnosis of myocardial infarction was verified by a cardiologist.

\section{Statistical analysis}

Continuous variables are presented as mean $\pm \mathrm{SD}$ or median and IQR, and categorical variables as counts with proportions. Comparison of between-group continuous variables was performed using the unpaired t-test, whereas the Pearson $\chi^{2}$, two-tailed test was used for comparison of categorical variables.

A survival analysis was conducted with individual subject time-to-MACE using the Kaplan-Meier method, with curves representing MACE-free survival according to zero CAC or non-zero CAC. Annualised rates of MACE and all-cause mortality were estimated by dividing the number of events by the number of person-years at risk.

HR and 95\% CIs were calculated with the Cox proportional hazards regression model using zero CAC as the reference group. Three models were constructed: Model 1: unadjusted, univariable analysis; Model 2: multivariable analysis adjusting for age and gender; Model 3: multivariable analysis adjusting for age, gender and cardiovascular risk factors (hypertension, diabetes, obesity, smoking history and family history).

All statistical analyses were performed using IBM SPSS Statistics for Macintosh, V.25.0. A two-tailed $p$ value of $<0.05$ was considered statistically significant.

\section{RESULTS}

\section{Characteristics of Study cohort}

Over a 7-year period, 2253 patients underwent cardiac CT imaging at our institution. In all, 500 patients were excluded from the study cohort as they underwent imaging for indications other than investigation of chest pain, or because of a non-diagnostic calcium scan. Therefore, 1753 patients with symptomatic chest pain (mean age $56.8 \pm 12.0$ years) were included in the study 


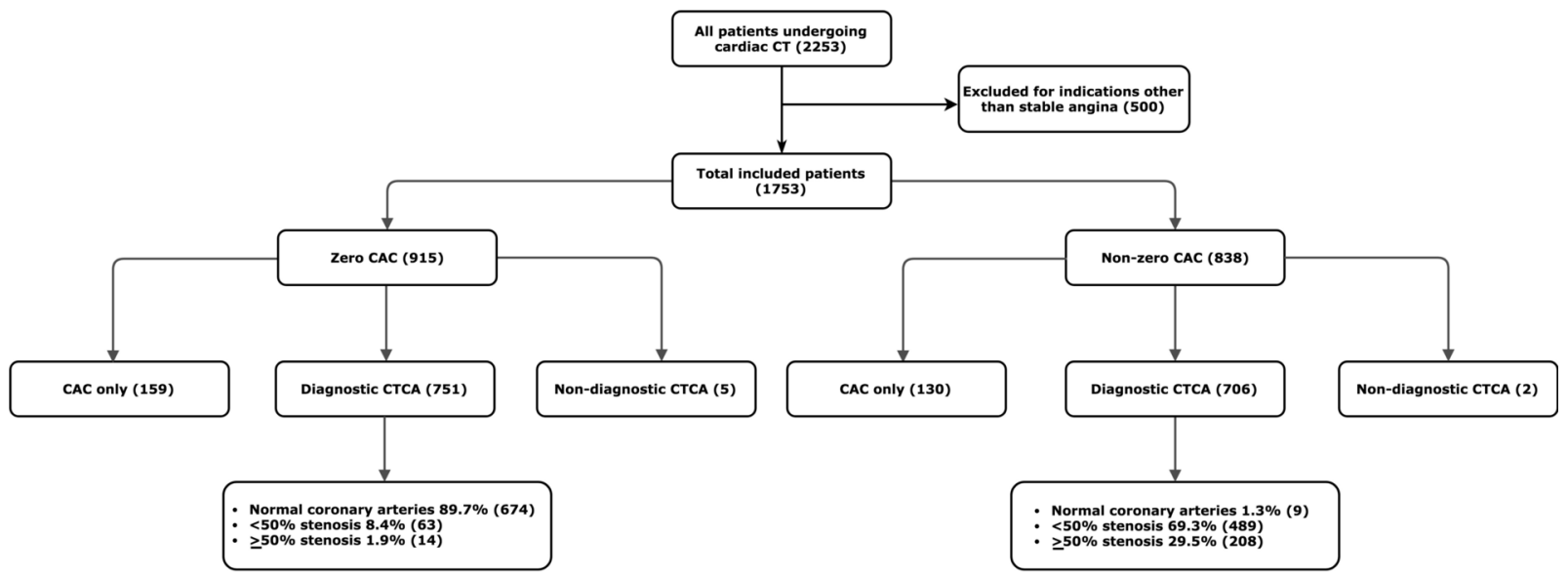

Figure 1 Study flow chart. The values in parentheses represent the numbers of patients in each part of the study. The percentages in the lowest boxes represent the fraction of patients in each disease category. CAC, coronary artery calcification; CTCA, CT coronary angiography.

(figure 1), and $46.2 \%$ were men. $52.2 \%$ of patients had a zero CAC versus $47.8 \%$ with non-zero CAC scores (median score 57.4 Agatston units; IQR 8.2-221). Patients with no detectable CAC were statistically more likely to be younger, women and less likely to have hypertension, diabetes or have a positive smoking history (table 1).

A total of 289 patients did not undergo CTCA after CAC scoring (159 patients with a zero CAC and 130 with non-zero CAC). The decision to perform a CTCA after CAC scoring was in line with contemporaneous clinical guidelines and hospital protocol. The major reasons were first, a low pretest probability for CAD plus a zero CAC score $(\mathrm{n}=112)$, and second, extensive calcification on CAC scan thought likely to preclude CTCA analysis $(n=88)$. CTCA was not performed in a further 89 patients because of difficult venous access, contrast allergy or patient preference. In addition, subjects with non-interpretable CTCA scans $(n=7)$ were excluded from the CAD extent analysis, but their calcium scores and clinical progress contributed to MACE and mortality estimates.

Of the 751 patients with a zero CAC in whom CTCA was performed, $674(89.7 \%)$ had normal coronary arteries, $63(8.4 \%)$ had $<50 \%$ stenosis and $14(1.9 \%)$ had $\geq 50 \%$ stenosis (figure 2). In the non-zero CAC cohort, of the 706 patients who underwent CTCA, 9 (1.3\%) had normal coronary arteries, 489 (69.3\%) had $<50 \%$ stenosis and $208(29.5 \%)$ had $\geq 50 \%$ stenosis.

In those with a zero CAC, there was no significant difference in the baseline risk factors in those with or without obstructive CAD ( $\geq 50 \%$ stenosis) with the exception of obesity and smoking history (table 2 ).

The negative predictive value of a zero CAC for excluding obstructive CAD ( $\geq 50 \%$ stenosis) was $98.1 \%$. The sensitivity of this was $93.7 \%$ and the specificity was $59.7 \%$.

\section{Follow-up and MACE}

Patients were followed up for a median of 2.2 years (IQR 1.8-3.7 years). The longest follow-up period was 7 years. Kaplan-Meier curves for MACE-free survival are presented in figure 3. The number of MACEs was $5(0.6 \%)$ in the zero CAC cohort versus $17(2 \%)$ in the non-zero CAC cohort. The annualised rate of MACE in the zero CAC cohort was 1.9 per 1000 person-years versus 7.4 per 1000 person-years in the non-zero CAC cohort (table 3).

MACE in those with zero CAC were due to non-fatal myocardial infarction and/or non-elective revascularisation. None died of a coronary event. MACE occurred at a time interval of 13 days to 1.8 years after cardiac CT. Of the patients who had a zero CAC, three patients had

\begin{tabular}{lllll}
\hline Table 1 & \multicolumn{3}{l}{ Baseline characteristics of all patients and stratified according to CAC score } & \\
\hline Variable & $\begin{array}{l}\text { All patients } \\
(\mathbf{n = 1 7 5 3 )}\end{array}$ & $\begin{array}{l}\text { CAC=0 } \\
(\mathbf{n}=\mathbf{9 1 5})\end{array}$ & $\begin{array}{l}\text { CAC }>\mathbf{0} \\
(\mathbf{n}=\mathbf{8 3 8})\end{array}$ & P value \\
\hline Age (years), mean \pm SD & $56.8 \pm 12.0$ & $51.7 \pm 11.1$ & $62.3 \pm 10.5$ & $<0.0001$ \\
\hline Male gender, N (\%) & $810(46.2 \%)$ & $360(39.3 \%)$ & $450(53.7 \%)$ & $<0.0001$ \\
\hline Hypertension, N (\%) & $564(32.3 \%)$ & $198(21.6 \%)$ & $366(43.7 \%)$ & $<0.0001$ \\
\hline Diabetes mellitus, N (\%) & $135(7.7 \%)$ & $40(4.4 \%)$ & $95(11.3 \%)$ & $<0.0001$ \\
\hline Obesity, N (\%) & $211(12.0 \%)$ & $98(10.7 \%)$ & $113(13.5 \%)$ & 0.0746 \\
\hline Smoking history, N (\%) & $462(26.4 \%)$ & $219(23.9 \%)$ & $243(29.0 \%)$ & 0.0162 \\
\hline Family history, N (\%) & $559(31.9 \%)$ & $300(32.8 \%)$ & $259(30.9 \%)$ & 0.3989 \\
\hline
\end{tabular}

CAC, coronary artery calcification; $\mathrm{N}$, number. 


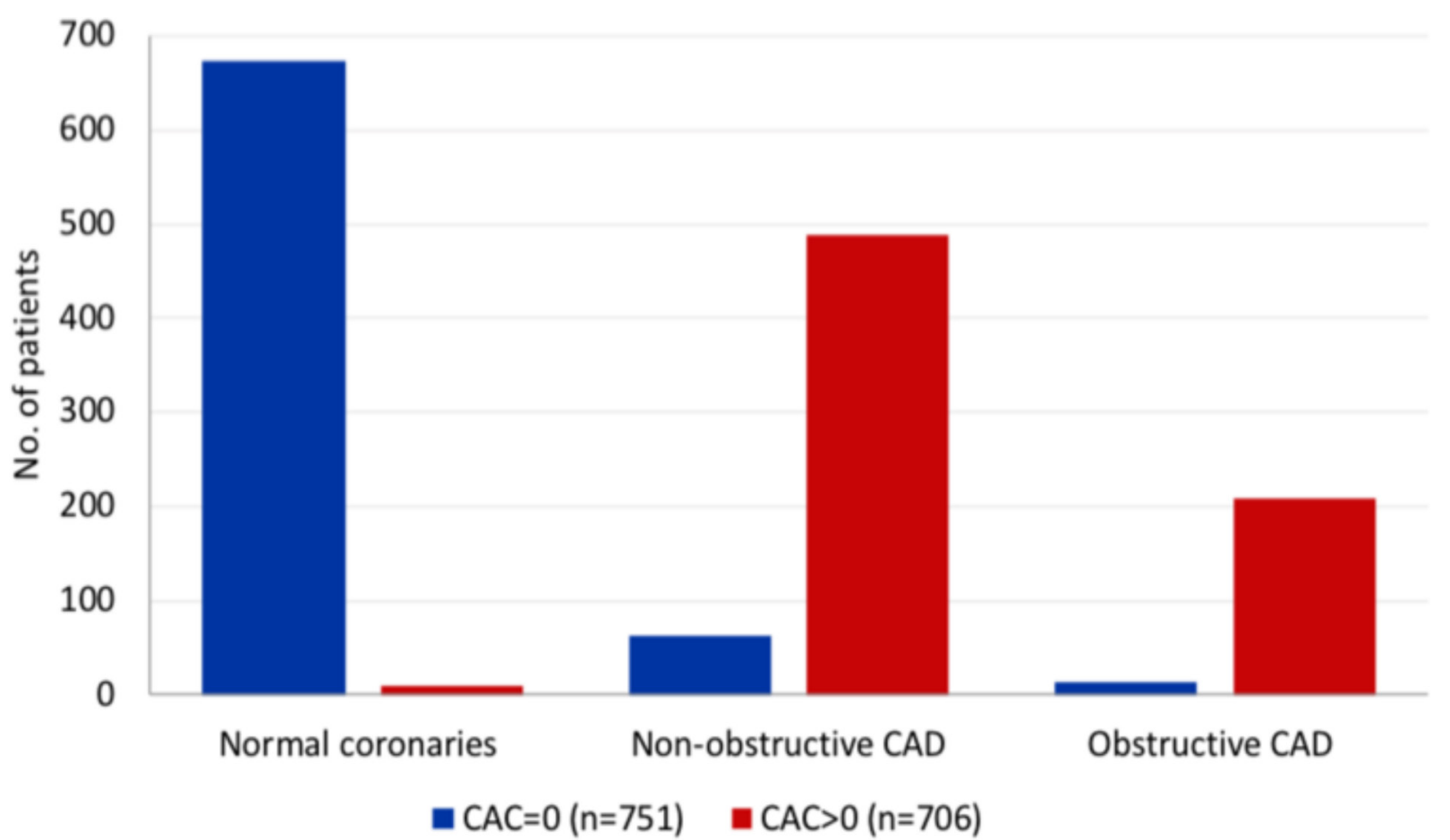

Figure 2 Prevalence of CAD following CTCA stratified according to CAC score. CAC, coronary artery calcification; CAD, coronary artery disease; CTCA, CT coronary angiography.

normal coronary arteries and one patient had $\geq 50 \%$ stenosis on CTCA. Of note, 1 patient who had a zero CAC score did not have CTCA and subsequently developed a non-ST elevation myocardial infarction and received non-elective revascularisation at 116 days post-scan.

In the unadjusted univariable analysis, there was moderate evidence that the survival distributions for the zero CAC versus the non-zero CAC cohort differed (HR $3.8,95 \%$ CI 1.4 to $10.3, \mathrm{p}=0.009)$. After adjusting for the confounding factors age and gender, this difference was no longer statistically significant (HR 2.6, 95\% CI 0.87 to $7.9, \mathrm{p}=0.09$ ). After adjusting for age, gender plus cardiovascular risk factors, the HR for MACE among the zero CAC cohort vers non-zero CAC was 2.1 (95\% CI 0.69 to $6.5, \mathrm{p}=0.19)$.

Table 2 Baseline characteristics of patients with a zero CAC and absence or presence of $\geq 50 \%$ stenosis on CTCA

\begin{tabular}{llll}
\hline Variable & $\begin{array}{l}\text { CAD }<50 \% \\
(\mathbf{n = 7 3 7 )}\end{array}$ & $\begin{array}{l}\text { CAD } \geq 50 \% \\
(\mathbf{n = 1 4 )}\end{array}$ & P value \\
\hline Age (years), mean \pm SD & $51.73 \pm 11.24$ & $54.49 \pm 8.13$ & 0.3619 \\
\hline Male gender, N (\%) & $311(42.2 \%)$ & $8(57.1 \%)$ & 0.2624 \\
\hline Hypertension, N (\%) & $166(22.5 \%)$ & $5(35.7 \%)$ & 0.2436 \\
\hline Diabetes mellitus, N (\%) & $30(4.1 \%)$ & $1(7.1 \%)$ & 0.5670 \\
\hline Obesity, N (\%) & $71(9.6 \%)$ & $4(28.6 \%)$ & 0.0192 \\
\hline Smoking history, N (\%) & $165(22.4 \%)$ & $8(57.1 \%)$ & 0.0022 \\
\hline Family history, N (\%) & $230(31.2 \%)$ & $6(42.9 \%)$ & 0.3523 \\
\hline
\end{tabular}

CAC, coronary artery calcification; CAD, coronary artery disease;CTCA, CT coronary angiography; N, number.

\section{All-cause mortality}

During follow-up, the incidence of all-cause mortality was three patients $(0.17 \%)$ in the zero CAC cohort versus 20 patients $(1.1 \%)$ in the non-zero CAC cohort. Annualised rates of all-cause mortality for the zero CAC cohort were 1.2 per 1000 person-years vers 8.7 per 1000 person-years in the non-zero CAC cohort.

In the unadjusted univariable analysis of all-cause mortality, there was evidence that the survival distributions for the zero CAC versus the non-zero CAC cohort differed (HR 7.6, 95\% CI 2.3 to 26, $\mathrm{p}=0.001$ ). After adjusting for age and gender, this was no longer statistically significant (HR 2.5, 95\% CI 0.67 to $9.1, \mathrm{p}=0.18$ ). After adjusting for age, gender plus cardiovascular risk factors, the HR for all-cause mortality among the zero CAC cohort versus non-zero CAC was 2.1 (95\% CI 0.56 to $8.0, \mathrm{p}=0.27$ ).

\section{Composite endpoint: MACE and/or all-cause mortality}

A composite endpoint comprising non-fatal myocardial infarction, non-elective revascularisation and/or all-cause mortality resulted in seven patients $(0.40 \%)$ in the zero CAC cohort versus 36 patients $(2.05 \%)$ in the non-zero CAC cohort with events. Following univariable analysis, there was evidence that the survival distributions for the zero CAC versus the non-zero CAC cohort differed (HR 5.8, 95\% CI 2.6 to 13.0, $\mathrm{p}<0.0001$ ). This difference remained statistically significant after adjusting for age and gender (HR 2.8, 95\% CI 1.2 to $6.8, \mathrm{p}=0.02$ ). After adjusting for age, gender plus cardiovascular risk factors, this was no longer statistically significant (HR 2.3, 95\% CI 0.9 to $5.7, \mathrm{p}=0.065)$. 


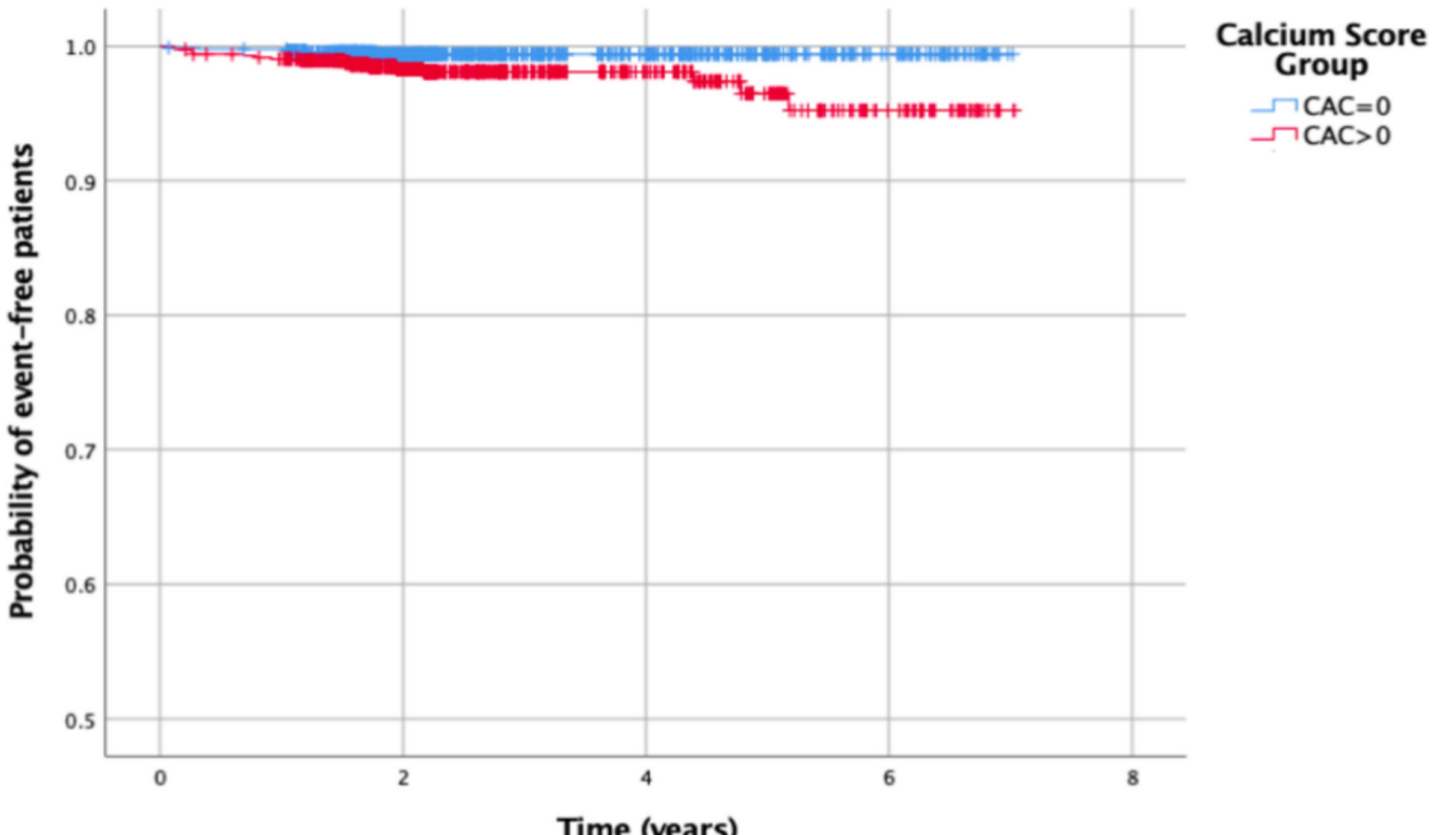

Number at risk
\begin{tabular}{|c|c|c|c|c|c|c|c|}
\hline Time (years) & $\mathbf{0}$ & $\mathbf{1}$ & $\mathbf{2}$ & $\mathbf{3}$ & $\mathbf{4}$ & $\mathbf{5}$ & $\mathbf{6}$ \\
\hline CAC $=0$ & 915 & 910 & 577 & 292 & 213 & 110 & 58 \\
\hline CAC > 0 & 838 & 822 & 524 & 243 & 165 & 93 & 46 \\
\hline
\end{tabular}

Figure 3 Kaplan-Meier curves stratified according to CAC score (total population). CAC, coronary artery calcification

\section{DISCUSSION}

Studies in asymptomatic individuals have shown that the absence of CAC is a reliable screening tool in the evaluation for suspected CAD. ${ }^{39}$ However, there is uncertainty in the prevalence of CAD in symptomatic patients with a zero CAC score and in its longer-term prognostic implications. $^{10}$

\section{Prevalence of non-calcified CAD in symptomatic patients with a zero CAC}

In this prospective cohort study of patients with stable chest pain, a zero calcium score was associated with a low rate of $\mathrm{CAD}$, with $8.4 \%$ subjects having non-obstructive disease and only $1.9 \%$ found to have obstructive disease. Furthermore, a zero score had a high negative predictive value of $98.1 \%$ for excluding obstructive CAD.

Table 3 Annualised MACE rates for all patients and stratified according to CAC score

\begin{tabular}{lccl}
\hline & $\begin{array}{l}\text { No. of } \\
\text { patients (\%)* }\end{array}$ & $\begin{array}{l}\text { No. of } \\
\text { MACE (\%)† }\end{array}$ & $\begin{array}{l}\text { Rate/1000 } \\
\text { person-years at } \\
\text { risk }\end{array}$ \\
\hline CAC $=0$ & $915(52.2 \%)$ & $5(0.55 \%)$ & 1.92 \\
CAC $>0$ & $838(47.8 \%)$ & $17(2.03 \%)$ & 7.37 \\
All patients & $1753(100 \%)$ & $22(1.25 \%)$ & 3.46 \\
\hline
\end{tabular}

*Proportion of all patients.

†Proportion within each group.

.CAC, coronary artery calcification; MACE, major adverse cardiac event.
Our results agree with similar studies in symptomatic patient populations. Among patients with zero CAC in the COroNary CT Angiography Evaluation For Clinical Outcomes: An InteRnational Multicenter Registry (CONFIRM) registry, $13 \%$ had at least one $<50 \%$ stenosis and $3.5 \%$ had at least one $\geq 50 \%$ stenosis. ${ }^{10}$ In a singlecentre prospective cohort study of 1114 patients, the prevalence of $\geq 50 \%$ stenosis was $4.3 \% .{ }^{11}$ Negative predictive values of $99.5 \%, 99 \%$ and $96 \%$, respectively, have been quoted in the literature, ${ }^{1012} 13$ close to our findings. The negative predictive value for zero CAC compares well with CTCA and stress imaging tests. ${ }^{14}$

\section{Prognostic significance of a zero CAC}

Over a medium-term follow-up, the incidence of MACE and all-cause mortality in our patients was lower in the zero CAC cohort than the non-zero CAC cohort. In multivariable survival analysis after adjusting for age, gender and cardiovascular risk factors, there was no difference in the prognosis between the two cohorts for both MACE and all-cause mortality end points $(\mathrm{p}=0.19$ and $\mathrm{p}=0.27$, respectively). However, the overall prognosis of stable angina in patients with zero CAC was good with an annualised rate of MACE in the zero CAC cohort of 1.9 per 1000 person-years.

We compared both MACE and all-cause mortality in patients with zero CAC versus non-zero CAC, whereas previous studies have focused only on all-cause mortality, and showed this to be $0.05 \%$ to $3.8 \%$ over a mean follow-up period of 5.6-15 years. ${ }^{3912}$ In another study, the 
incidence of MACE was found to be $1.3 \%$ over 2.8 years. ${ }^{11}$ Importantly, these studies align with our findings that the overall prognosis of patients with a zero CAC is largely unaffected by the presence of non-calcified atheroma. ${ }^{12}$

\section{CAC scoring versus CTCA}

Although subjects with zero CAC had a good prognosis over during medium-term follow-up, $10.3 \%$ were found to have CAD on subsequent CTCA. Once identified, this group can benefit prognostically from medical therapy as demonstrated in the Scottish COmputed Tomography of the HEART Trial (SCOTHEART). ${ }^{15} 16$ The discovery of underlying $\mathrm{CAD}$ in those with zero calcium scores highlights the advantages of using CTCA in all subjects rather than having calcium scoring as a gatekeeper, as was the case in the previous version of the NICE CG95 guideline. CTCA without prior CAC scoring also lowers patient radiation exposure.

\section{Study limitations}

As an observational study, clinical data were obtained from the electronic records system and there may have been incomplete patient follow-up. We assumed that loss to follow-up would occur equally across the study cohort, thereby not unduly influencing results. As a single-centre study, our findings may not be applicable in other geographic regions. Finally, while we analysed the degree of luminal stenosis on CTCA, we did not take into account the impact of high-risk plaque features that are also known to have prognostic significance. ${ }^{17}$

\section{CONCLUSION}

A zero CAC score in patients with stable chest pain reliably excludes obstructive $\mathrm{CAD}$ and is associated with an overall very good prognosis. However, CTCA provides further diagnostic and prognostic information, revealing underlying $\mathrm{CAD}$ in over $10 \%$ of patients. We now know that such patients should be treated with preventive medical therapy to improve their prognosis.

Contributors Contributors XW and EPVL contributed equally to the data collection, the statistical analysis and drafted the initial manuscript. JMT, NJHP and NKR contributed to the data collection. HP advised on the statistical analysis. JHFR, JB, MW, JMT and DG planned the study and JHFR is the study guarantor.

Funding JHFR is part-supported by the National Institute of Health Research (NIHR) Cambridge Biomedical Research Centre, the Higher Education Funding Council for England, the British Heart Foundation, the EPSRC and the Wellcome Trust. EPVL is funded by the Frank Edward Elmore Fund and the Medical Research Council's Doctoral Training Partnership. JMT is supported by the Wellcome Trust.

Competing interests None declared.

Patient consent for publication Not required.

Ethics approval The study was a planned clinical service evaluation and was carried out after favourable review by the hospital's audit board.
Provenance and peer review Not commissioned; externally peer reviewed.

Data sharing statement No additional data are available.

Open access This is an open access article distributed in accordance with the Creative Commons Attribution 4.0 Unported (CC BY 4.0) license, which permits others to copy, redistribute, remix, transform and build upon this work for any purpose, provided the original work is properly cited, a link to the licence is given, and indication of whether changes were made. See: https://creativecommons.org/ licenses/by/4.0/.

\section{REFERENCES}

1. Budoff MJ, McClelland RL, Nasir K, et al. Cardiovascular events with absent or minimal coronary calcification: the multi-ethnic study of atherosclerosis (MESA). Am Heart J 2009;158:554-61.

2. Moss AJ, Williams MC, Newby DE, et al. The updated NICE guidelines: cardiac CT as the first-line test for coronary artery disease. Curr Cardiovasc Imaging Rep 2017;10.

3. Valenti V, ó Hartaigh B, Heo R, et al. A 15-year Warranty period for asymptomatic individuals without coronary artery calcium. JACC Cardiovasc Imaging 2015;8:900-9.

4. Sarwar A, Shaw LJ, Shapiro MD, et al. Diagnostic and prognostic value of absence of coronary artery calcification. JACC Cardiovasc Imaging 2009;2:675-88.

5. Hulten E, Bittencourt MS, Ghoshhajra B, et al. Incremental prognostic value of coronary artery calcium score versus CT angiography among symptomatic patients without known coronary artery disease. Atherosclerosis 2014;233:190-5.

6. Rosen BD, Fernandes V, McClelland RL, et al. Relationship between baseline coronary calcium score and demonstration of coronary artery stenoses during follow-up MESA (multi-ethnic study of atherosclerosis). JACC Cardiovasc Imaging 2009;2:1175-83.

7. National Institute for Health and Care Excellence. Chest pain of recent onset: assessment and diagnosis (clinical guidance 95), 2016 1-16. Available: https://www.nice.org.uk/guidance/cg95[Accessed 2 Apr 2018].

8. Agatston AS, Janowitz WR, Hildner FJ, et al. Quantification of coronary artery calcium using ultrafast computed tomography. J Am Coll Cardiol 1990;15:827-32.

9. Blaha M, Budoff MJ, Shaw LJ, et al. Absence of coronary artery calcification and all-cause mortality. JACC Cardiovasc Imaging 2009;2:692-700.

10. Villines TC, Hulten EA, Shaw LJ, et al. Prevalence and severity of coronary artery disease and adverse events among symptomatic patients with coronary artery calcification scores of zero undergoing coronary computed tomography angiography. J Am Coll Cardiol 2011;58:2533-40.

11. Kim YJ, Hur J, Lee H-J, et al. Meaning of zero coronary calcium score in symptomatic patients referred for coronary computed tomographic angiography. Eur Heart J Cardiovasc Imaging 2012;13:776-85.

12. Mittal TK, Pottle A, Nicol E, et al. Prevalence of obstructive coronary artery disease and prognosis in patients with stable symptoms and a zero-coronary calcium score. Eur Hear J Cardiovasc Imaging 2017;18:922-9.

13. Abdool MA, Ashrafi R, Davies M, et al. A UK cardiac centre experience of low-risk, stable chest pain patients with calcium score of zero. Br J Cardiol 2014;21.

14. Meijboom WB, Meijs MFL, Schuijf JD, et al. Diagnostic accuracy of 64-slice computed tomography coronary angiography: a prospective, multicenter, multivendor study. J Am Coll Cardiol 2008;52:2135-44.

15. Adamson PD, Hunter A, Williams MC, et al. Diagnostic and prognostic benefits of computed tomography coronary angiography using the 2016 National Institute for health and care excellence guidance within a randomised trial. Heart 2018;104:207-14.

16. The SCOT-HEART Investigators. Coronary CT angiography and 5-year risk of myocardial infarction. N Engl J Med 2018;379:924-33.

17. Motoyama S, Ito $\mathrm{H}$, Sarai $\mathrm{M}$, et al. Plaque characterization by coronary computed tomography angiography and the likelihood of acute coronary events in mid-term follow-up. J Am Coll Cardiol 2015;66:337-46. 